\title{
Characterization of candidate genes for bovine adipogenesis reveals differences of TUSC5 isoforms caused by novel alternative splicing
}

\author{
Yang Zhou ${ }^{1}$, Mingxun $\mathrm{Li}^{1,2}$, Xinde Hu${ }^{3,4}$, Hanfang Cai ${ }^{1}$, Liushuai Hua ${ }^{1,5}$, Jing Wang ${ }^{1,5}$, \\ Yongzhen Huang ${ }^{1}$, Xianyong Lan ${ }^{1}$, Chuzhao Lei ${ }^{1}$, George E. Liu ${ }^{6}$, Congjun Li ${ }^{6}$, Martin \\ Plath $^{1}$ and Hong Chen ${ }^{1}$ \\ ${ }^{1}$ College of Animal Science and Technology, Northwest A\&F University, Shaanxi Key Laboratory of Agricultural Molecular \\ Biology, Yangling, Shaanxi 712100, China \\ ${ }^{2}$ College of Animal Science and Technology, Yangzhou University, Yangzhou, Jiangsu 225009, China \\ ${ }^{3}$ Institute of Neuroscience, Chinese Academy of Sciences, Shanghai 200031, China \\ ${ }^{4}$ College of Veterinary Medicine, Northwest A\&F University, Yangling, Shaanxi 712100, China \\ ${ }^{5}$ Institute of Animal Husbandry and Veterinary Science, Henan Academy of Agricultural Sciences, Henan Key Laboratory of Animal \\ Breeding and Nutritional Regulation, Zhengzhou, Henan 450002, China \\ ${ }^{6}$ Animal Genomics and Improvement Laboratory, BARC, USDA-ARS, Beltsville, Maryland 20705, USA \\ Correspondence to: Hong Chen, email: chenhong1212@263.net
}

Keywords: cattle, lipid accumulation, TUSC5, alternative splicing, subcellular localization

Received: August 22, $2017 \quad$ Accepted: October 05, $2017 \quad$ Published: January 02, 2018

Copyright: Zhou et al. This is an open-access article distributed under the terms of the Creative Commons Attribution License 3.0 (CC BY 3.0), which permits unrestricted use, distribution, and reproduction in any medium, provided the original author and source are credited.

\section{ABSTRACT}

Different transcripts generated by alternative splicing have led to new insights to reconsider gene functions. Here, by progressively screening $\mathbf{3 1}$ candidate genes, we detected 3 genes that could be regulated by the peroxisome proliferator activated receptor gamma 2 (PPARG2), one isoform of the PPARG that specifically expressed in adipose tissue. Alternative splicing events of two genes regulated by PPARG2 cell death-inducing DFFA-like effector c (CIDEC) and tumor suppressor candidate 5 (TUSC5) - were further investigated. Similar results regarding their subcellular localization were observed for two isoforms of CIDEC. We validated the existence and coding ability of a novel TUSC5 transcript (TUSC5a). Differences became apparent between the two TUSC5 transcript isoforms in terms of expression and subcellular localization, possibly caused by a 29 amino acid insertion. The expression of the TUSC5a was significantly delayed, and showed that uniquely expressed in adipose tissue and differently expressed with TUSC5b during adipocyte differentiation. Subcellular localization analyses showed that both TUSC5 isoforms existed in the endplasmic reticulum but with different localization and no interaction with CIDEC isoforms. In summary, our candidate gene-based approach provides further depth to our understanding of the process of adipogenesis, highlighting the functional diversity of one gene generated by alternative splicing.

\section{INTRODUCTION}

Mammalian adipogenesis - the formation of matured adipocytes from precursors - is regulated by complex molecular mechanisms [1]. On the one hand, scientific interest in the process of adopogenesis is fuelled by the fact that obesity, caused by unusually high lipid deposition, has become a concern for human health worldwide. Obesity can lead to numerous serious diseases, such as diabetes, hypertension, and various forms of cancer [2-5]. On the other hand, the content of fat tissues in skeletal muscles plays a key role in determining meat (e.g., beef) palatability, textural quality, and flavor [6, 7]. A considerable number of genes related to adipogenesis have been characterized so far 
[8]. However, it became apparent that transcript isoforms, generated from the same gene through alternative splicing, provide another level of complexity when trying to unravel the regulatory mechanisms underlying adipogenesis [9].

Studies on alternative splicing explore the vast proteomic complexity seen in higher eukaryotes, while typically focusing on a limited number of candidate genes [10-11]. Based on NGS deep sequencing methodologies, current estimates suggest that around $95 \%$ of human genes with multiple exons produce at least two transcript isoforms $[12,13]$. The diversity introduced by alternative splicing highlights the need to explore the biological function(s) of multiple transcript isoforms. Indeed, an increasing number of studies exemplify how alternative isoforms can be functionally very different. For example, alternative splicing of the stromal interaction molecule 2 (STIM2) gene converts the function of the respective proteins from an activator to an inhibitor of store-operated calcium channels [14]. Oligonucleotideinduced alternative splicing of the serotonin $2 \mathrm{C}$ receptor (HTR2C) was found to reduce food intake in mice [15]. In humans, analyses of protein interaction networks including isoforms arising from alternative splicing found that the majority of isoform pairs shared less than $50 \%$ of interaction partners, and interaction partners that were specific to alternative isoforms tended to be expressed in a highly tissue-specific manner and belong to distinct functional modules [16]. Altogether then, studies considering potential novel/additional functions of isoforms arising from alternative splicing are highly warranted even for well characterized genes [17].

Studies on mammalian adipogenesis have long established that one of the key regulators of adipogenesis, PPARG, exists in two isoforms in adipocytes, which arise from differential promoter usage of the PPARG gene [1820]. PPARG1 is widely expressed in different tissues, while PPARG2 is uniquely expressed in adipose tissue [19]. A PPARG knockdown assay also revealed that PPARG2, but not PPARG1, reactivated adipogenesis [17]. Subsequently, several other genes related to adipogenesis or adipocyte differentiation were reported. For example, only the small soluble isoform of the delta-like non-canonical Notch ligand $1(D l k 1)$ can inhibit adipocyte differentiation in mice [21]. Moreover, Sam68 was reported to regulate alternative splicing of the ribosomal protein S6 kinase (S6K1) during adipogenesis in mice [22]. In humans, alternative splicing events through the actions of the transformer 2 beta homolog $(T R A 2 B)$, BCL2 associated athanogene 6 (BAG6), and mutS homolog 5 (MSH5) contribute to transcriptomic and proteomic diversity and could play important roles in regulating obesity [23]. Still, a number of mammalian genes with alternative transcript isoforms need to be characterized regarding their (regulatory) functions.

The quality of beef is largely dependent on the intramuscular fat content. Our understanding of the regulatory mechanisms involved in bovine adipogenesis is clearly limited compared to studies on adipogenesis in humans or other model organisms. Moreover, the regulation of alternative splicing is highly variable among species [24]. In this study, to identify genes functioning during adipogenesis of cattle and clarify the differences between different isoforms of the genes, we concentrated on a set of 31 candidate genes obtained from previous RNA sequencing studies in cattle and examined the functions of alternative splicing variants [25]. We found 12 genes to be robustly expressed in bovine adipose tissue. Two genes (CIDEC and TUSC5) were up-regulated by PPARG2 and were selected for further study. We found no difference between alternative splicing isoforms in their subcellular localization in the case of CIDEC. By contrast, the two transcript isoforms of TUSC5 showed different expression patterns and different localizations in the smooth endplasmic reticulum. We discuss how both isoforms could regulate adipogenesis without interacting with CIDEC. Our study supplies resources of candidate genes for adipogenesis and promotes the understanding of the effects of alternative splicing on gene functions.

\section{RESULTS}

\section{Selection of highly expressed candidate genes in bovine adipose tissue}

One set of candidate genes that could play roles in fat deposition were selected according to a previous study using RNA sequencing for transcriptomic profiling of bovine adipose tissue [25]. We compared expression levels of two development stages (fetal and adult bovine adipose tissues) and selected 11 candidate genes based on the following criteria: (a) expression levels exceeding 10 reads per kilobase of transcript per million mapped reads (RPKM) and $(b)$ expression levels in adult adipose tissue being at least 10 times higher than in fetal tissue (Supplementary Table 1). Second, we selected another 20 genes that were highly and commonly expressed (all > 500 RPKM) in various samples of bovine adipose tissues (fetal, as well as adult cow, bull and steer; Supplementary Table 1). We used quantitative PCR (qPCR) to quantitatively compare expression levels of the 31 genes between different tissues: heart, liver, spleen, lung, kidney, fat tissue, skeletal muscle, stomach, and intestine of adult Qinchuan cows (Figure 1A and Supplementary Figure 1). We thus obtained eight genes (TUSC5, SCD, CIDEC, THRSP, LIPE, S100B, GHR and $A D I P O R 2)$ from the first type and four (SPARC, CST3, $A N X A 2$ and $V I M$ ) from the second type of candidate genes that were robustly expressed in adipose tissue.

\section{Screening genes related to adipogenesis and regulated by PPARG2}

No study has yet reported different isoforms of the bovine $P P A R G$ gene, and so we first confirmed the existence 
and coding sequences of bovine PPARG1 and PPARG2 isoforms. We successfully cloned the two transcripts of the bovine PPARG gene corresponding to the sequences NM_181024.2 and BC116098.1 in the GenBank database (Supplementary Figure 2B). The NM_181024.2 sequence was found to code for an additional $3 \overline{0}$ amino acids in the 5'-region of the coding region contained in BC116098.1 (Supplementary Figure 2A). By consulting previous studies [26] and using blast searches against murine PPARG1 and PPARG2 sequences, we established that the BC116098.1 sequence codes for bovine PPARG1, while the NM_181024.2 sequence codes for bovine PPARG2 (Supplementary Figure 2C and Supplementary Figure 2D).

Rosiglitazone, an agonist of PPARG, was used to induce bovine adipocyte adipogenesis for 2, 4 and 6 days and to examine the expression of the 12 candidate genes robustly expressed in adult adipose tissues (i.e., during lipid accumulation). Lipid droplets appeared after two days of induction and became larger by day 4 and day 6 (Supplementary Figure 3). Expression of PPARG1 increased significantly during day 2 and decreased again on day 4 and day 6. PPARG2 expression also increased on day 2 , remained stable during the next two days, and increased significantly again on day 6 . We used following thresholds to select genes for further analysis: (1) the gene expression increased in all the day 2, day 4 and day 6 comparing to day $0 ;(2)$ the gene expression increased significantly $(p<0.05)$ comparing to day 0 in at least one time point of day 2 , day 4 and day 6 . Finally, seven genes (GHR, THRSP, SCD, LIPE, TUSC5, CIDEC and CST3) met the above criteria that had increased expression levels after lipid droplets appeared (Figure 1B).

Previous studies found that unlike PPARG1, PPARG2 was exclusively expressed in adipose tissue [27]. We over-expressed bovine PPARG2 in bovine adipocytes using an adenovirus and examined gene expression after 2 days (Figure 1C). The expression of PPARG1 did not change $(p>0.05)$ while PPARG2 was significantly overexpressed $(p<0.05)$, corroborating that the results of our study specifically depict effects caused by PPARG2 and downstream activated genes. We found three genes (CIDEC, TUSC5 and CST3) to be significantly upregulated $(p<0.05)$, probably mediated by PPARG2.

\section{Alternative splicing variants of $C I D E C$ and TUSC 5 transcripts}

All three genes that were up-regulated by PPARG2 had more than one transcript in our RNA sequencing dataset [25] or in the GenBank database, suggesting alternative splicing events. We selected the cell death inducing DFFAlike effector c $(C I D E C)$ and tumor suppressor candidate 5 (TUSC5) genes for further study. In total, we found four different transcripts (XM_010817501.2, XM_005222498.3, NM_001076031.1 and XM_015459442.1) of the CIDEC gene. Considering only coding regions, three different isoforms were detected and henceforth referred to as CIDEC1 (XP_015314928.1), CIDEC2 (NP_001069499.1) and CIDEC3 (XP_005222555.1, XP_010815803.1). Only one transcript of the bovine TUSC5 gene (NM_001083707.1) was annotated in GenBank. We did, however, retrieve one alternative splicing event from our previous RNA sequencing data-set, with $87 \mathrm{nt}$ in the 5'-end of the third exon alternatively inserted between the second and third exons (Figure 2A). The novel TUSC5 transcript was confirmed in adipose tissues from three different cows by PCR amplification from cDNA and Sanger sequencing (Figure 2B). The $87 \mathrm{nt}$ insertion encodes 29 amino acids with no frame shift or premature translational termination (Figure 2C). Thus, the novel TUSC5 transcript includes three exons and codes for 206 amino acids. The novel splicing site of TUSC5 also conformed to the GT-AG principle at exon-intron boundaries. The two transcripts of the bovine TUSC5 gene are henceforth referred to as TUSC5a (novel GenBank accession number: KP347628.1) and TUSC5b (NM_001083707.1).

\section{Different expression profiles of TUSC5a and TUSC5b}

We compared the expression profiles of the two TUSC5 transcript isoforms in different bovine tissues and at different stages of adipogenesis using qPCR. TUSC5a was unique to adipose tissue (Figure 3A), while TUSC5b was highly expressed in adipose tissue but could also be detected in other tissues, such as heart and skeletal muscle tissue (Figure 3A). The expression of total TUSC5 (i.e., both isoforms) increased by 70 -fold (compared to its abundance on day 0 ) on 2 days after differentiation was induced and increased to 340-fold during the following 8 to 10 days. This pattern was largely driven by changes in TUSC5b expression, whereas changes in TUSC5a expression were much weaker. TUSC5a increased app. 18fold after 2 days followed by a continuous decrease during the following 4 to 6 days, and increased to 30 -fold during the 8 days and 10 days comparing to 0 day.

\section{In vitro expression and translation analyses of TUSC5a}

We confirmed that the novel TUSC5 transcript can be translated into a protein by cloning it into the pDSred-N1 vector and transfecting it into 293T cells, using TUSC5b as a positive control. TUSC5a was translated in the form of a fusion protein. However, the TUSC5a fusion protein showed obviously translational retardation compared to that of the TUSC $5 \mathrm{~b}$ fusion protein even though the same dose of pDSred-N1-TUSC5a and pDSred-N1-TUSC5b were used to transfect the 293T cells using highly standardized procedures (Supplementary Figure 4). The TUSC5b fusion protein could be detected already 12 hours after transfection, while the TUSC5a 
fusion protein required almost another 12 hours to emerge. After 48 hours, the TUSC5b fusion protein became abundant (as evidenced by considerable brightness of the fluorescent signal; Supplementary Figure 4), while the TUSC5a fusion protein was still less abundant (i.e. lighter fluorescent signal; Supplementary Figure 4). We also examined mRNA expression levels of TUSC5a and TUSC $5 \mathrm{~b}$ at different points in time after transfection (Figure 3C). Both mRNA expression levels (TUSC5a and TUSC5b) reached peaks within the first 24 hours. However, throughout the experiment, expression levels of TUSC5a were significantly lower than those of TUSC5b.

\section{Comparing the intracellular localizations of CIDEC and TUSC5 transcript isoforms}

We examined the localization of two CIDEC transcript isoforms (CIDEC2, CIDEC3) and both TUSC5 transcript isoforms (TUSC5a, TUSC5b) by separately cloning them into the pDSred-N1 vector (red fluorescence) or the pEGFP-C1 vector (green fluorescence) and cotransfecting 3T3-L1 cells with the respective pair of isoforms. We first examined whether the differently fused tags from pDSred-N1 and pEGFP-C1 could affect the intracellular localization of the resulting proteins. However, we found almost complete congruence of the localizations of different fusion proteins containing CIDEC3 and TUSC5b (Figures 3D, Supplementary Figure 5).

Both isoforms of CIDEC and TUSC5 were found in the cytoplasma. No obvious difference was observed between the intracellular localizations of CIDEC2 and CIDEC3 (Supplementary Figure 5). By contrast, TUSC5a and TUSC5b appeared to be differently distributed (only partially overlapped between the fluorescent signals of TUSC5a and TUSC5b; Figure 3D). In the case of TUSC5b, we identified a cellular location signal in the C-terminal

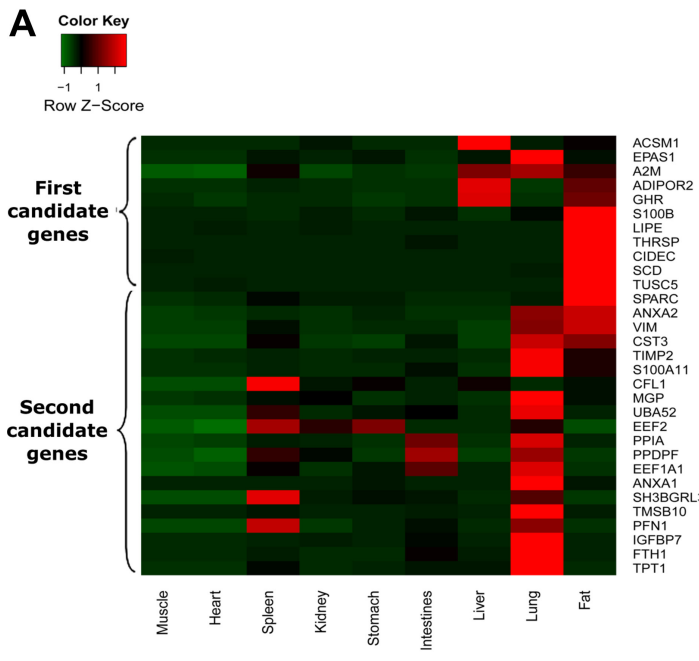

B
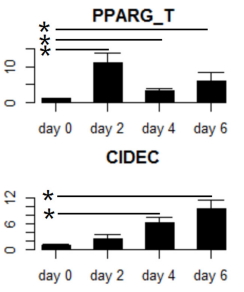

day 0 day 2 day 4 day GHR

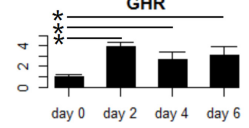

THRSP

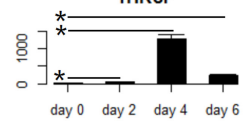
ADIPOR2

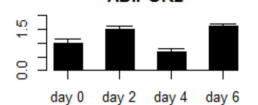

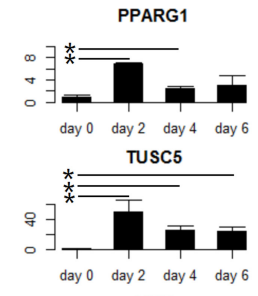

LIPE

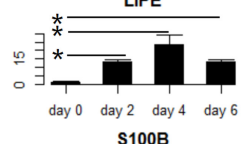

S100B

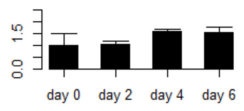

SPARC

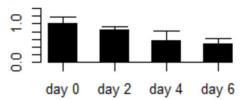

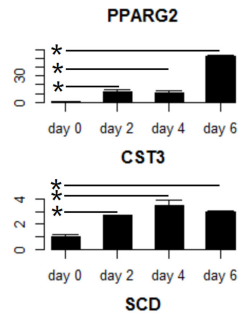

$\operatorname{SCD}$
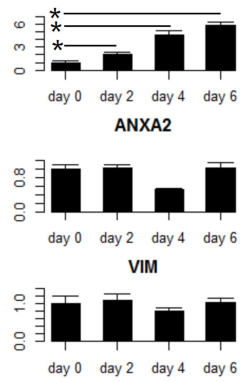
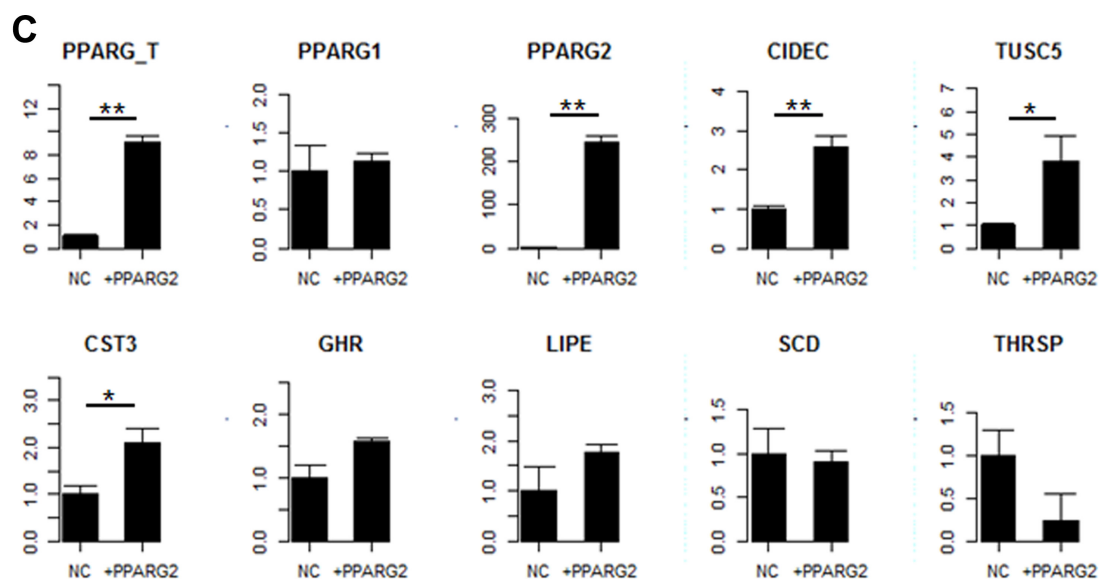

Figure 1: Screening candidate genes for the bovine adipogenesis gene. (A) A summary for the qPCR results of the 31 candidate gene using heatmap plot and detail information can be seen in Supplementary Figure 1. (B) displays candidate genes' expression changes during adipogenesis induced by rosiglitazone. The genes with increased expression level in day 2, day 4 and day 6 were compared to day 0 and were tested for significant differences. ${ }^{*} P<0.05$. (C) shows candidate genes' expression changes induced by PPARG2. The y axis of both Figure 1B and Figure 1C showed relative mRNA abundance. ${ }^{*} P<0.05 ;{ }^{* *} P<0.01$. 
end (https://wolfpsort.hgc.jp/), which was altered by the 29 amino acid insertion seen in TUSC5a. For both isoforms we predicted endplasmic reticulum (ER) membrane retention signals (KKXX-like motifs: TVPK for TUSC5a and AVPK for TUSC 5b). The change between threonine (T) and alanine (A) could be responsible for the observed differences in subcellular localization between TUSC5a and TUSC5b.

\section{Subcellular localization analysis of TUSC5 isoforms}

We conducted analyses of co-localization of TUSC5a or TUSC5b focusing on the Golgi apparatus, the rough ER, and the smooth ER separately in 3T3-L1 cell lines. The TUSC5a and TUSC5b fusion proteins showed overlap in their occurrence in the smooth ER - the site of cellular lipid (fat) and steroid hormone production - and in the rough ER - the site of protein biosynthesis (Figure 4A, 4B). Strong accumulation of both isoforms in the smooth ER is consistent with their predicted functions during lipid deposition.
As both TUSC5 and CIDEC were strongly expressed during adipogenesis, we next examined whether they might interact. We selected CIDEC2 to perform co-localization with TUSC5a and TUSC5b, using the pDSred-N1 or pEGFP-C1 vectors, separately. However, the results showed that the subcellular localization of CIDEC2 overlapped with neither TUSC5a nor TUSC5b, rendering interactions unlikely (Figure 5). We examined the subcellular localization of CIDEC2 in the Golgi apparatus, as well as in the rough and smooth ER, and inside mitochondria. However, CIDEC2 was found in none of the organelle (Supplementary Figure 6).

\section{DISCUSSION}

In our study, we found the expression of seven genes to be significantly up-regulated after treatment with a PPARG agonist, but not all of them showed consistent results when we over-expressed PPARG2 in bovine adipocytes. This may be due to several factors:

A

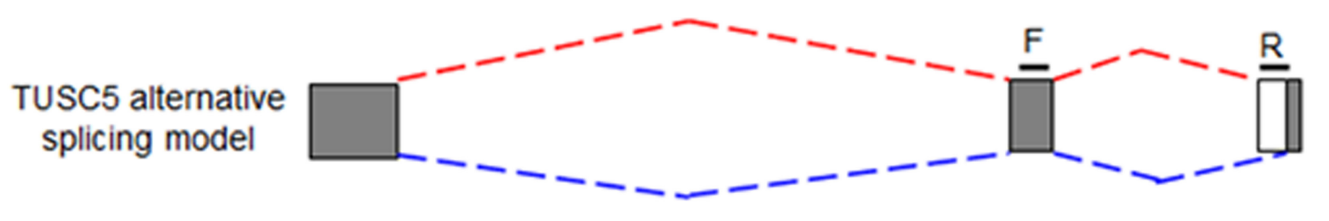

B
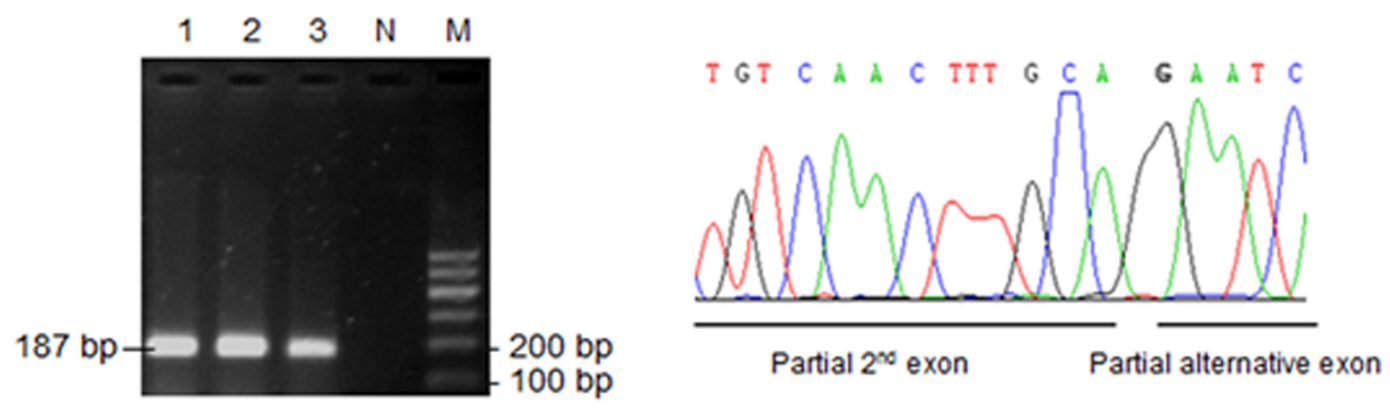

C

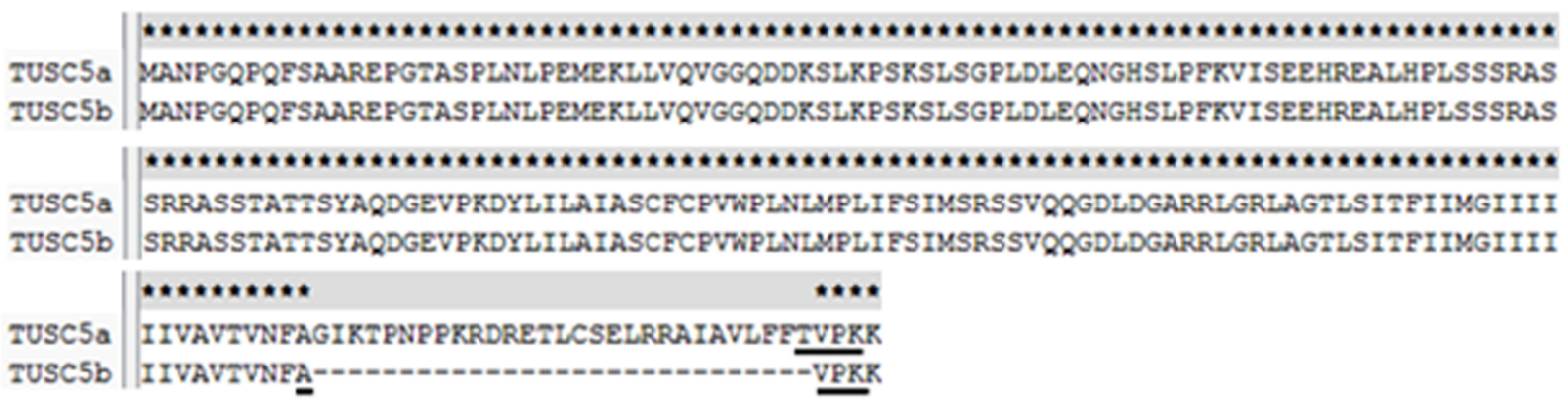

Figure 2: Validation of the alternative splicing of bovine TUSC5. (A) TUSC5 alternative splicing patterns and primer positions for validating TUSC5a. The grey boxes are the known exons; the white box is the alternative spliced exon. The red and blue imaginary lines represent two different exon combination models. F and R represents the forward and reverse primers. (B) Agrose gel electrophoresis result and sanger sequencing result for amplifying the partial TUSC5a. The 1-3 lanes are the amplification result for the cDNA from 3 cows' adipose tissues, N: Negative control, M: Marker. (C) The alignment between the amino acid sequences of TUSC5a and TUSC5b. The amino acid sequences with underline were retention signals predicted by a website tool (https://wolfpsort.hgc.jp/). 
(i) the PPARG agonist could also affect other pathways, (ii) the respective gene was up-regulated by the effect of other gene products or regulatory elements, (iii) lipid deposition was induced directly by the PPARG agonist, or (iv) PPARG1 and PPARG2 target different genes. Rosiglitazone could influence cell signaling through additional mechanisms, such as the induction of a number of signaling intermediates number of signaling intermediates $[28,29]$. In this study, we did not examine whether or not PPARG1 and PPARG2 target different genes, but previous studies have highlighted obvious functional differences between the two PPARG isoforms
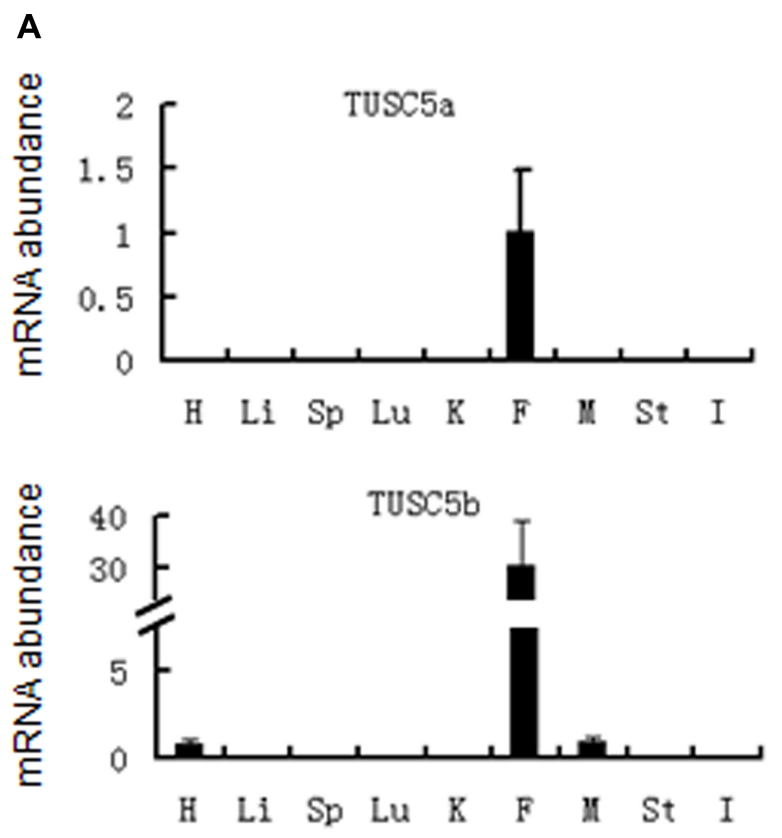

D
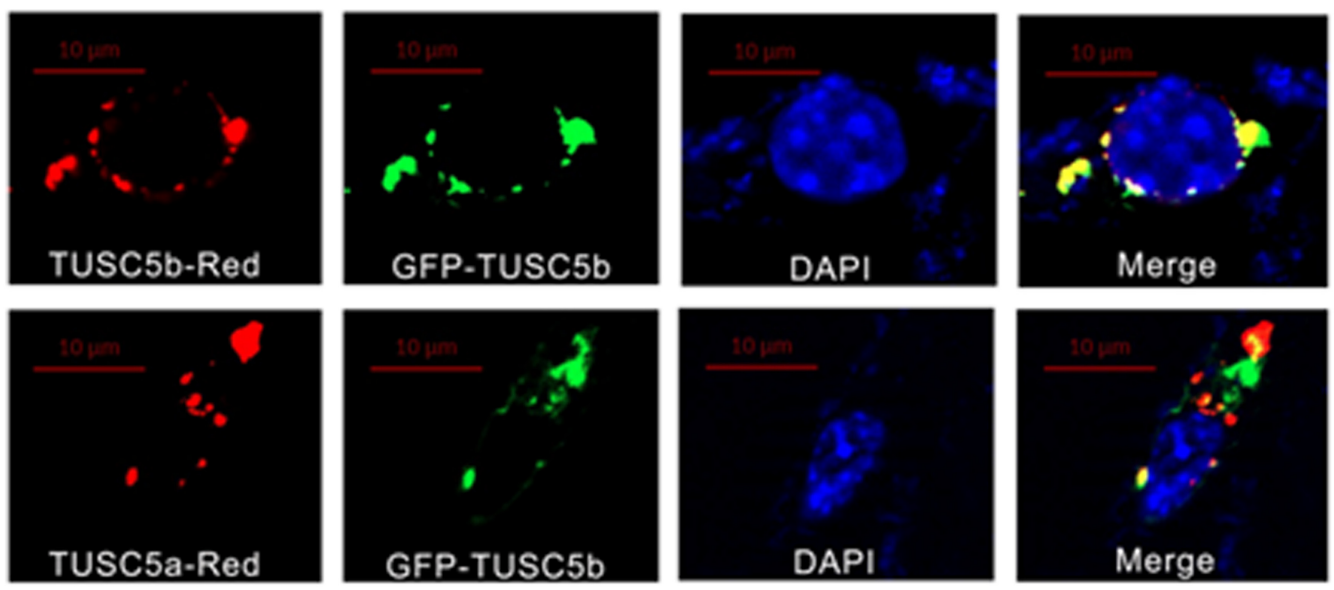

Figure 3: Expression and intracellular localization differences between the TUSC5 isoforms. (A) Tissue expression profiles for bovine TUSC5a and TUSC5b. H: heart; Li: Liver; Sp: spleen; Lu: lung; K: kidney; F: fat tissue; M: muscle; St: stomach; I: intestines. (B) The expression changes of different transcripts of TUSC5 during differentiation of ADSCs. (C) The mRNA expression of TUSC5apDSred and TUSC5b-pDSred in 293T cell line of different time; the expression level were evaluated using qPCR. ${ }^{*} P<0.05 ;{ }^{* *} P<0.01$. (D) Cellular localization analysis of TUSC5a and TUSC5b. 

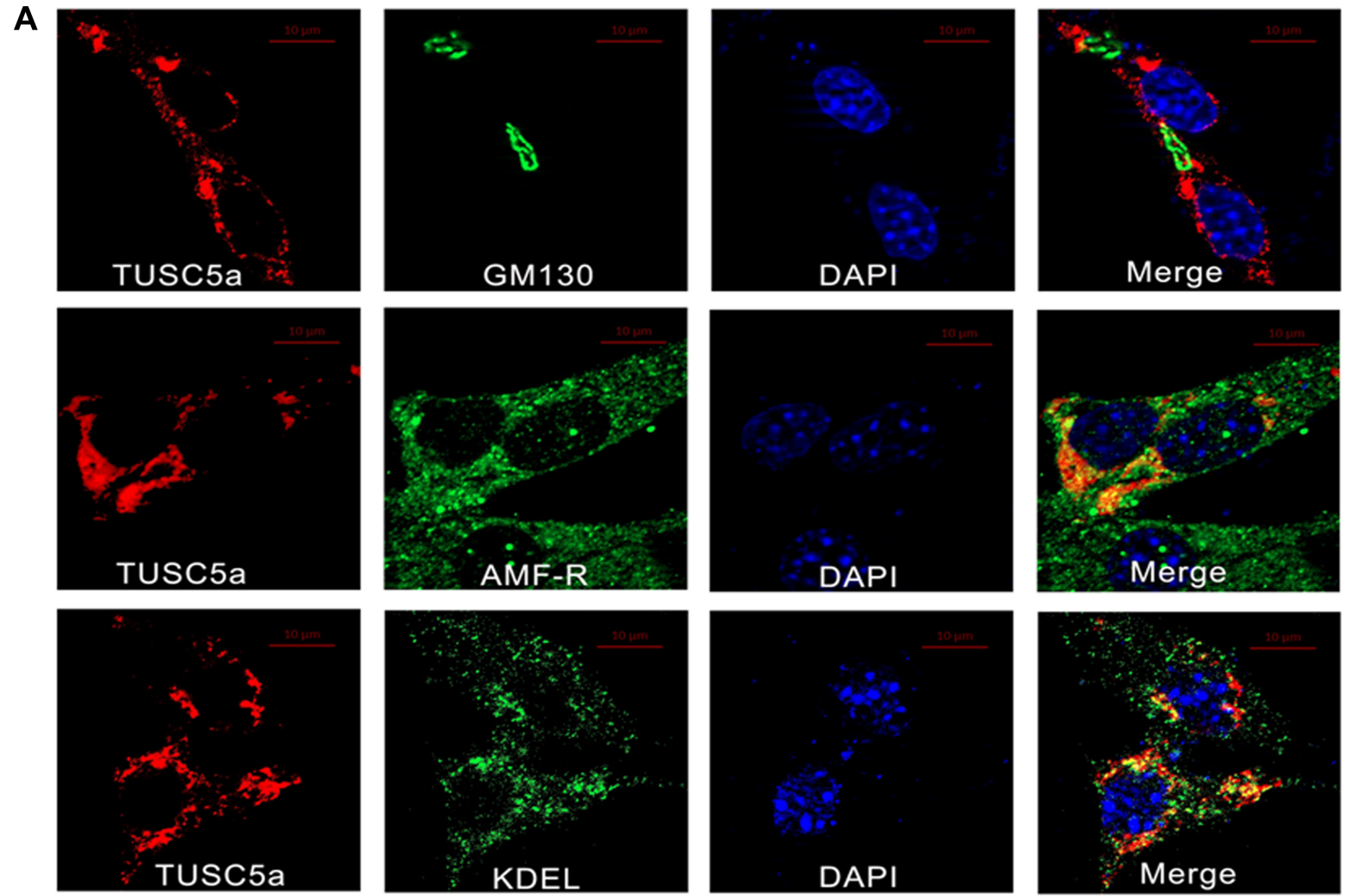

B
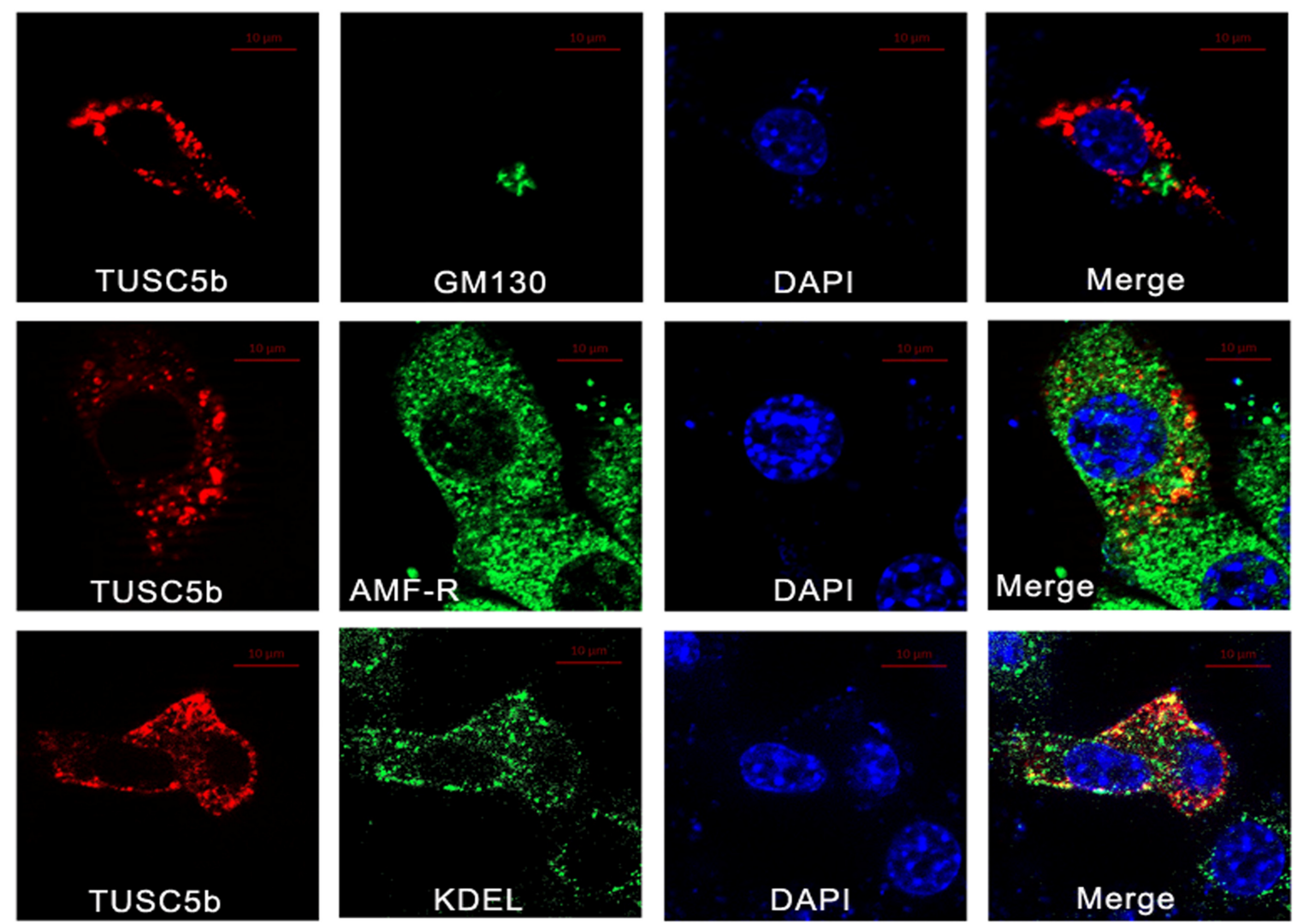

Figure 4: Subcellular localization analysis of TUSC5a and TUSC5b. (A) Subcellular localization analysis of TUSC5a in 3T3-L1 cells; (B) Subcellular localization analysis of TUSC5b in 3T3-L1 cells. The antibodies used in this study were GM130 for golgi, KDEL for rough ER, AMF-R for smooth ER. The nuclei were stained by DAPI. 
gene promoter region, but we retrieved a binding site in the CCAAT/enhancer-binding protein alpha $(C E B P a)$ promotor region that could interact with PPARG, with a positive cross-regulation loop [31]. Therefore, both TUSC5 and CST3 could be regulated by PPARG2 during adipogenesis, and future research will need to concentrate on the questions of what the exact targets of both PPARG1 and PPARG2 might be.

We focused on two genes likely to be targeted by PPARG2 (CIDEC and TUSC5), both of which show alternative splicing events. CIDEC was reported to play vital roles in adipogenesis [30, 32-34]. TUSC5 was reported to show robust expression in both white and brown adipose tissues and research has started to draw attention to TUSC5 as a novel regulator of insulin action in adipocytes in studies on mice and humans [29, 35-37]. Our present study is the first to investigate potential functional differences between different TUSC5 transcript isoforms. The cellular localization of CIDEC2 and CIDEC3 were almost perfectly overlapped in the cytoplasm, leaving the possibility that they might have similar functions. However, obvious differences were observed between TUSC5a and TUSC5b in terms of their expression profiles and their intracellular localization.

Alternative sequence insertion or deletion can lead to various alterations of gene functions, depending on the splicing site(s); on the other hand, gene functions may be not changed if the functional domain is not affected. We predicted the protein structure of CIDEC2 and CIDEC3, using SMART online tools (http://smart.embl-heidelberg. $\mathrm{de} /$ ). No structural difference was found between the two isoforms. However, one amino acid was substituted in the ER membrane retention signals of both TUSC5 isoforms. We found their intracellular localizations (especially in the smooth, and to a lesser extent in the rough ER) to be slightly different, suggesting that they may have different functions. Beside potential structure changes, alternative splicing may produce isoforms with different dosages of expression. In our study, we failed to amplify CIDEC1 cDNA, which may be due to exceedingly low mRNA abundance. Of both TUSC5 isoforms, TUSC5b was more representative of total TUSC5 mRNA content in the expressional profiles than TUSC5a, suggesting that TUSC5b was more expressed. Moreover, TUSC5b transcripts emerged earlier when comparing the expression of both isoforms of the TUSC 5 gene during adipocyte differentiation. Altogether then, TUSC5b appears to be the dominant transcript of the bovine TUSC5 gene. Both isoforms may have similar functions, act cooperatively, independently, or even conversely [38]. Future studies will need to elaborate on the potentially divergent function(s) of both bovine TUSC 5 gene isoforms.

Both TUSC5 and CIDEC were robustly expressed in adipose tissue, which led to the hypothesis that they might interact. Considering intracellular localizations, we excluded the possibility of specific fusion tags effecting the localizations of the respective proteins. The CIDEC protein is involved in cell apoptosis [39], and accordingly, we observed condensation in the nucleus 48 hours after transfection into $293 \mathrm{~T}$ cells, which showed easier apoptosis than 3T3-L1 cells in our experiment [40]. Therefore, the function of CIDEC was likely not affected by the fusion tags. Importantly, we did not observe spatial overlap between their fusion proteins regarding their intracellular localization: while both TUSC5 isoforms were observed especially in the smooth ER, CIDEC was not detected in the golgi apparatus, rough and smooth ER, or in mitochondria. A previous study found that CIDEC was enriched in lipid droplet-contacting sites and promoted lipid transfer from smaller to larger lipid droplets [41]. CIDEC participated in the lipid synthesis process after lipids were produced. TUSC5, on the
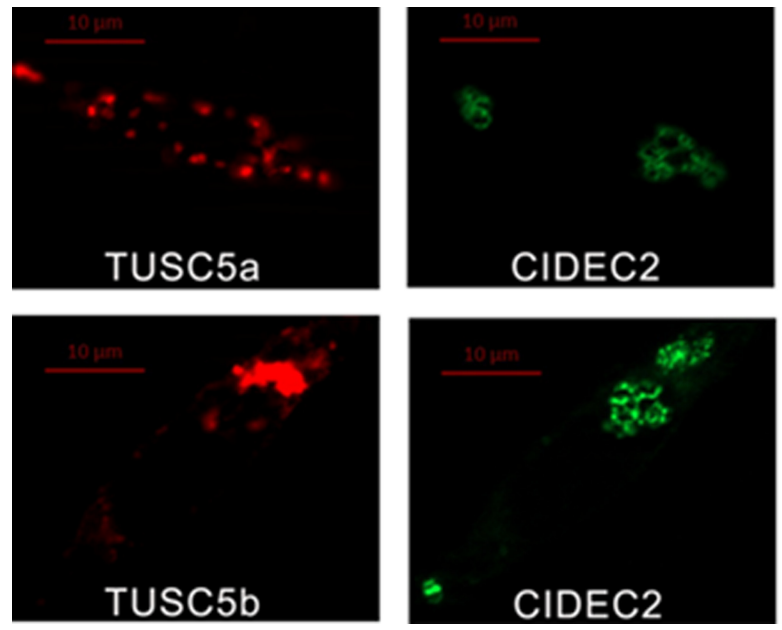
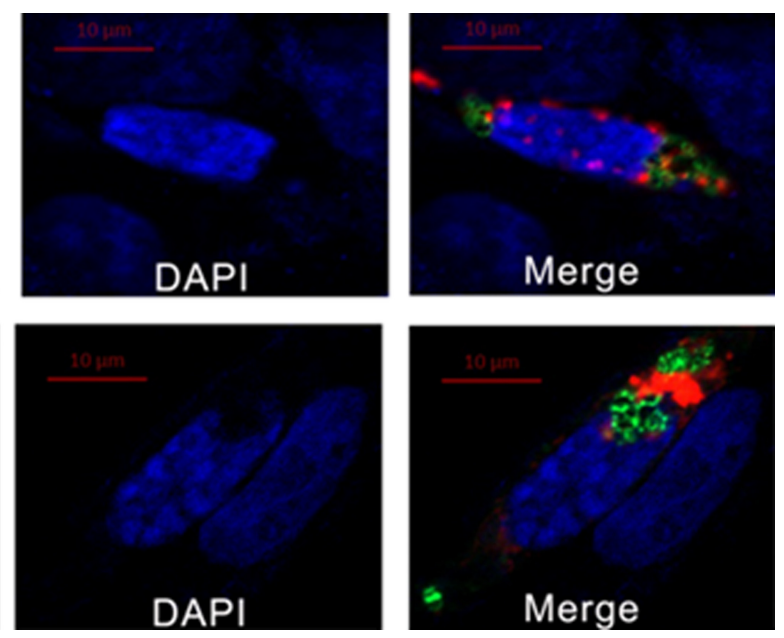

Figure 5: Co-localization analysis of TUSC5a, TUSC5b and CIDEC2. First rows: co-localization analysis of pDSred-N1TUSC5b and pEGFP-C1 -TUSC5b in 3T3-L1 cells. Second rows: co-localization analysis of pDSred-N1-TUSC5a and pEGFP-C1TUSC5b in 3T3-L1 cells. Third rows: co-localization analysis of pDSred-N1-TUSC5a and pEGFP-C1-CIDEC2 in 3T3-L1 cells. Fourth rows: co-localization analysis of pDSred-N1-TUSC5b and pEGFP-C1-CIDEC2 in 3T3-L1 cells. The nuclei were stained by DAPI. 
other hand, was found to positively regulate the insulinstimulated glucose transport in adipocytes [29]. Given that the smooth ER is responsible for producing lipids, we argue that TUSC5 is involved in regulating liponeogenesis without interacting with CIDEC.

Our study detected potentially functional differences between the two isoforms of TUSC5. However, we did not find alternative splicing reports for TUSC5 gene in human and mouse. The alternative splicing has been proved that can be diversity among different species (Pan et al. 2005). Thus our study will not only help to understand the effects of alternative splicing on TUSC5 gene functions in cattle but also promote the further detection of different isoforms of TUSC5 gene in other species or the specific functions of the TUSC5a if the alternative splicing is only happened in cattle.

\section{MATERIALS AND METHODS}

\section{Tissue collection}

All animals used in this study were approved by the Institutional Animal Care and Use Committee of Northwest A \& F University and Qinbao Animal Husbandry Co., Ltd, respectively. The methods used in this study were performed in accordance with the relevant guidelines in the Shaanxi Key Laboratory of Agricultural Molecular Biology of Northwest A\&F University. Different tissues (heart, liver, spleen, lung, kidney, fat, muscle, stomach, and intestines) were collected from adult Qinchuan cows (female and aged from 20 to 24 months) immediately after being slaughtered, snap-frozen in liquid nitrogen, and stored at $-80^{\circ} \mathrm{C}$ until use.

\section{Bovine adipocyte culture, rosiglitazone induction, and PPARG2 over-expression}

Bovine adipocyte-derived stem cells (ADSCs) were isolated and cultured as we described previously [42]. The ADSCs were plated in 6-well plates (NUNC) containing $1.5 \mathrm{ml}$ Dulbecco's modified Eagle's medium (DMEM) supplemented with $10 \%$ fetal bovine serum, $100 \mu \mathrm{g} / \mathrm{ml}$ streptomycin, and $100 \mathrm{U} / \mathrm{ml}$ penicillin at $37^{\circ} \mathrm{C}$ with a $\mathrm{CO}_{2} 5 \%$ atmosphere. Cell culture media was replaced every two days. Rosiglitazone (Sigma), at a concentration of 1,000 nmol/L was used for inducing adipogenesis. The cells were collected every two days (48 hours) from day 0 to day 6 . Adenoviruses with the bovine PPARG2 gene (supplied by Liushuai Hua and Jing Wang, Institute of Animal Science, Henan Academy of Agricultural Sciences, Henan 450000, China) were used for over-expressing PPARG2 in bovine adipocytes. The infected cells were collected after culturing for two days to extract total RNAs.

\section{RNA isolation and qPCR}

Total RNA was extracted from each tissue or collected cells using the Trizol method, according to the manufacturer's instructions (TaKaRa). Total RNA $(1.0 \mu \mathrm{g})$ was reverse-transcribed to cDNA using the PrimeScript RT reagent kit with gDNA Eraser. qPCR was performed using a Bio-Rad CFX 96 Real Time Detection System and SYBR Green PCR Master Mix in a $20 \mu$ reaction with three or four replicates. Primers for qPCR were designed using the NCBI primer-BLAST webtool (https://www. ncbi.nlm.nih.gov/tools/primer-blast/index.cgi?LINK $\mathrm{LOC}=\mathrm{BlastHome})$. The primer information was presented in Supplementary Table 2. ACTB was used as reference gene to calculate the relative gene expression values using the $2^{-\Delta \Delta \mathrm{Ct}}$ method.

\section{Gene cloning and vectors preparation}

Primers for gene cloning and vector construction were designed using the NCBI primer-BLAST webtool. Restriction enzyme cutting sites and the necessary nucleotides, avoiding coding frame shift for gene expression vector construction, were added manually. The detailed information for primers was presented in Supplementary Table 3.

PPARG1 was cloned using PPARG1-pcDNA-F and PPARG-pcDNA-R as primers. PPARG2 was cloned using PPARG2-pcDNA-F and PPARGpcDNA-R as primers. To validate the existence of alternative splicing event for the bovine TUSC5, we designed two specific primers (Figure 2A). The forward primer (TCTCGAAGTAGCGTGCAACA) was designed in the second exon and the reverse primer (GCTCAGAACACAGGGTCTCC) was designed in the alternative exon. PCR were performed in $25 \mu \mathrm{L}$ of reaction volume containing $1 \mu \mathrm{L}$ cDNA from adipose tissue, $1 \mu \mathrm{mol} / \mathrm{L}$ of each primer, $12.5 \mu \mathrm{L} 2 \times$ PrimeSTAR Max Premix (TaKaRa) for PPARG1 and PPARG2 cloning or $12.5 \mu \mathrm{L} 2 \times$ Taq MasterMix (TaKaRa) for validating the TUSC5 alternative splicing event. The PCR regimen for PPARG1 and PPARG2 cloning was as follows: initial denaturation for $5 \mathrm{~min}$ at $95^{\circ} \mathrm{C}$; followed by 35 cycles of $98^{\circ} \mathrm{C}$ for $10 \mathrm{~s}$; annealing at $59.5^{\circ} \mathrm{C}$ for $10 \mathrm{~s}$; and primer extension at $72^{\circ} \mathrm{C}$ for $1 \mathrm{~min}$. The final extension was performed at $72^{\circ} \mathrm{C}$ for $10 \mathrm{~min}$. The PCR regimen for validating the TUSC5 alternative splicing event was as follows: initial denaturation for $5 \mathrm{~min}$ at $95^{\circ} \mathrm{C}$; followed by 30 cycles of $95^{\circ} \mathrm{C}$ for $30 \mathrm{~s}$; annealing at $58^{\circ} \mathrm{C}$ for $30 \mathrm{~s}$; and primer extension at $72^{\circ} \mathrm{C}$ for $20 \mathrm{~s}$. The final extension was performed at $72^{\circ} \mathrm{C}$ for $5 \mathrm{~min}$. All the PCR reactions were carried out using a PCR System Thermal Cycler Dice (TaKaRa, Dalian, China).

The PCR products used to clone in pDSred-N1 vector or pEGFP-C1 vector for the different transcripts of TUSC5 gene and CIDEC gene were amplified using primers with specific restriction enzymes. The TUSC5a was cloned using overlap PCR strategy and other transcripts were used traditional PCR with same PCR reaction system as the PPARG transcript cloning. The primers and detail 
PCR amplification strategies can be see in Supplementary Table 3. The digested products were purified using DNA gel extraction kits (Sangon Biotech), legated using T4 DNA ligase (TaKaRa) at $16^{\circ} \mathrm{C}$ overnight and transformed into E.coli DH5 $\alpha$. Single clones were re-cultured, and the plasmids were isolated for sequencing identification.

\section{Gene expression and subcellular localization analysis}

Cells 293T and 3T3-L1 were cultured in DMEM, supplemented with $10 \%$ fetal bovine serum, $100 \mu \mathrm{g} / \mathrm{ml}$ streptomycin, and $100 \mathrm{U} / \mathrm{ml}$ penicillin at $37^{\circ} \mathrm{C}$ with $\mathrm{CO}_{2}$ $5 \%$ atmosphere. For the in vitro expression analysis of TUSC5 isoforms, $293 \mathrm{~T}$ cells cultured in 6-well plates (NUNC) and were transfected with $2500 \mathrm{ng}$ vectors with $5 \mu \mathrm{l}$ Liposomes 2000 (Invitrogen) for each well. For the subcellular localization analysis, 3T3-L1 cells were cultured in 24-well plates (NUNC) with seeded coverlips inside and transfected with $500 \mathrm{ng}$ vectors with $1 \mu \mathrm{l}$ Liposomes 2000 (Invitrogen) for each well. An equal number of vectors (250 ng for each) were used for the wells needed to perform co-localization analysis. After 24-48 hours, the cells were fixed using cold 4\% paraformaldehyde for $15 \mathrm{~min}$ and stained using DAPI (Millipore). The cells for subcellular localization analysis contained added antibody, and were diluted using PBS (Phosphate Buffered Saline), sitting overnight at $4^{\circ} \mathrm{C}$. The antibodies used in this study were mouse anti-GM130 (Sigma) for golgi, mouse anti-KDEL (Santa Cruz) for rough ER, anti-AMF-R (Santa Cruz) for smooth ER [43-45]. Next, FITC AffiniPure goat anti-mouse IgG (Jackson ImmunoResearch) and Alexa Fluor 568-conjugated donkey anti-mouse IgG (Life technology) were used for green and red fluorescence, separately. The mitochondria were stained using MitoTracker ${ }^{\circledR}$ Red CMXRos (Life technology). We washed the cells three times using PBS before and after each step. The fluorescent images were obtained using Zeiss microscope (Axio Observer Z1, ZEISS, Germany) at 630× amplification.

\section{Author contributions}

$\mathrm{YZ}$ conceived and designed the experiments. YZ, ML, XH, HC, LH and JW performed molecular biology analyses. YH, XL, CL and $\mathrm{CH}$ collected samples. GEL, $\mathrm{CL}, \mathrm{MP}$ and $\mathrm{YZ}$ wrote the paper.

\section{CONFLICTS OF INTEREST}

All authors declare no potential conflict of interest.

\section{FUNDING}

This study was supported by the National Natural Science Foundation of China (no. 31272408), the Program of National Beef Cattle and Yak Industrial Technology System (no. CARS-38), the Science and Technology Coordinator Innovative Engineering Projects of Shaanxi Province (2015KTCL02-08, 2014KTZB02-02-02-02), and the Bio-breeding Capacity-building and Industry-specific Projects from the National Development and Reform Commission (No. 2014-2573).

\section{REFERENCES}

1. Rosen ED, Walkey CJ, Puigserver P, Spiegelman BM. Transcriptional regulation of adipogenesis. Genes Dev. 2000; 14:1293-1307.

2. Mokdad AH, Ford ES, Bowman BA, Dietz WH, Vinicor F, Bales VS, Marks JS. Prevalence of obesity, diabetes, and obesity-related health risk factors, 2001. Jama. 2003; 289:76-79.

3. Rahmouni K, Correia ML, Haynes WG, Mark AL. Obesityassociated hypertension new insights into mechanisms. Hypertension. 2005; 45:9-14.

4. Hall JE, Hildebrandt DA, Kuo J. Obesity hypertension: role of leptin and sympathetic nervous system. Am J hypertension. 2001; 14:103S-115S.

5. Vucenik I, Stains JP. Obesity and cancer risk. evidence, mechanisms, and recommendations. Ann NY Acad Sci. 2012; 1271:37-43.

6. Wood J, Richardson R, Nute G, Fisher A, Campo M, Kasapidou E, Sheard P, Enser M. Effects of fatty acids on meat quality: a review. Meat Sci. 2004; 66:21-32.

7. Wood J, Enser M, Fisher A, Nute G, Sheard P, Richardson R, Hughes $\mathrm{S}$, Whittington F. Fat deposition, fatty acid composition and meat quality: A review. Meat Sci. 2008; 78:343-358.

8. Rankinen T, Zuberi A, Chagnon YC, Weisnagel SJ, Argyropoulos G, Walts B, Pérusse L, Bouchard C. The human obesity gene map: the 2005 update. Obesity. 2006; 14:529-644.

9. Péterfy M, Phan J, Reue K. Alternatively spliced lipin isoforms exhibit distinct expression pattern, subcellular localization, and role in adipogenesis. J Biol Chem. 2005; 280:32883-32889.

10. Dutertre M, Vagner S, Auboeuf D. Alternative splicing and breast cancer. RNA Biol. 2010; 7:403-411.

11. Luco RF, Allo M, Schor IE, Kornblihtt AR, Misteli T. Epigenetics in alternative pre-mRNA splicing. Cell. 2011; 144:16-26.

12. Pan Q, Shai O, Lee LJ, Frey BJ, Blencowe BJ. Deep surveying of alternative splicing complexity in the human transcriptome by high-throughput sequencing. Nat Genet. 2008; 40:1413-1415.

13. Wang ET, Sandberg R, Luo S, Khrebtukova I, Zhang L, Mayr C, Kingsmore SF, Schroth GP, Burge CB. Alternative isoform regulation in human tissue transcriptomes. Nature. 2008; 456:470-476.

14. Rana A, Yen M, Sadaghiani AM, Malmersjö S, Park CY, Dolmetsch RE, Lewis RS. Alternative splicing converts 
STIM2 from an activator to an inhibitor of store-operated calcium channels. J Cell Biol. 2015; 209:653-670.

15. Zhang Z, Shen M, Gresch PJ, Ghamari-Langroudi M, Rabchevsky AG, Emeson RB, Stamm S. Oligonucleotideinduced alternative splicing of serotonin $2 \mathrm{C}$ receptor reduces food intake. EMBO Mol Med. 2016; 8:878-894.

16. Yang X, Coulombe-Huntington J, Kang S, Sheynkman GM, Hao T, Richardson A, Sun S, Yang F, Shen YA, Murray RR. Widespread Expansion of Protein Interaction Capabilities by Alternative Splicing. Cell. 2016; 164:805-817.

17. Ren D, Collingwood TN, Rebar EJ, Wolffe AP, Camp HS. PPAR $\gamma$ knockdown by engineered transcription factors. exogenous PPAR $\gamma 2$ but not PPAR $\gamma 1$ reactivates adipogenesis. Genes Dev. 2002; 16:27-32.

18. Kawai M, Rosen CJ. PPAR $\gamma$ : a circadian transcription factor in adipogenesis and osteogenesis. Nat Rev Endocrinol. 2010; 6:629-636.

19. Fajas L, Auboeuf D, Raspé E, Schoonjans K, Lefebvre AM, Saladin R, Najib J, Laville M, Fruchart JC, Deeb S. The organization, promoter analysis, and expression of the human PPAR $\gamma$ gene. J Biol Chem. 1997; 272:18779-18789.

20. Lehrke M, Lazar MA. The many faces of PPAR $\gamma$. Cell. 2005; 123:993-999.

21. Baisong M, Ling Z, Li C, Sul HS. Only the large soluble form of preadipocyte factor-1 (Pref-1), but not the small soluble and membrane forms, inhibits adipocyte differentiation: role of alternative splicing. Biochem J. 2002; 364:137-144.

22. Song J, Richard S. Sam68 regulates S6K1 alternative splicing during adipogenesis. Mol Cell Biol. 2015; 35:1926-1939.

23. Kaminska D, Käkelä P, Nikkola E, Venesmaa S, Ilves I, Herzig KH, Kolehmainen M, Karhunen L, Kuusisto J, Gylling H. Regulation of alternative splicing in human obesity loci. Obesity. 2016; 24:2033-2037.

24. Laurell H, Grober J, Vindis C, Lacombe T, Dauzats M, Cecilia H, Langin D. Species-specific alternative splicing generates a catalytically inactive form of human hormonesensitive lipase. Biochem J. 1997; 328:137-143.

25. Zhou Y, Sun J, Li C, Wang Y, Li L, Cai H, Lan X, Lei C, Zhao X, Chen H. Characterization of transcriptional complexity during adipose tissue development in bovines of different ages and sexes. PloS one. 2014; 9:e101261.

26. Zhu Y, Qi C, Korenberg JR, Chen XN, Noya D, Rao MS, Reddy JK. Structural organization of mouse peroxisome proliferatoractivated receptor gamma (mPPAR gamma) gene: alternative promoter use and different splicing yield two mPPAR gamma isoforms. Proc Natl Acad Sci USA. 1995; 92:7921-7925.

27. Mueller E, Drori S, Aiyer A, Yie J, Sarraf P, Chen H, Hauser S, Rosen ED, Ge K, Roeder RG. Genetic analysis of adipogenesis through peroxisome proliferator-activated receptor $\gamma$ isoforms. J Biol Chem. 2002; 277:41925-41930.

28. Sugii S, Olson P, Sears DD, Saberi M, Atkins AR, Barish GD, Hong SH, Castro GL, Yin YQ, Nelson MC. PPAR $\gamma$ activation in adipocytes is sufficient for systemic insulin sensitization. Proc Natl Acad Sci USA. 2009; 106:22504-22509.

29. Fazakerley DJ, Naghiloo S, Chaudhuri R, Koumanov F, Burchfield JG, Thomas KC, Krycer JR, Prior MJ, Parker BL, Murrow BA. Proteomic analysis of GLUT4 storage vesicles reveals tumor suppressor candidate 5 (TUSC5) as a novel regulator of insulin action in adipocytes. J Biol Chem. 2015; 290:23528-23542.

30. Kim YJ, Cho SY, Yun CH, Moon YS, Lee TR, Kim SH. Transcriptional activation of Cidec by PPAR $\gamma 2$ in adipocyte. Biochem Biophys Res Commun. 2008; 377:297-302.

31. Lowell BB. PPARgamma: an essential regulator of adipogenesis and modulator of fat cell function. Cell. 1999; 99:239-242.

32. Shamsi BH, Ma C, Naqvi S, Xiao Y. Effects of pioglitazone mediated activation of PPAR- $\gamma$ on CIDEC and obesity related changes in mice. PLoS One. 2014; 9:e106992.

33. Rubio-Cabezas O, Puri V, Murano I, Saudek V, Semple RK, Dash S, Hyden CS, Bottomley W, Vigouroux C, Magré J. Partial lipodystrophy and insulin resistant diabetes in a patient with a homozygous nonsense mutation in CIDEC. EMBO Mol Med. 2009; 1:280-287.

34. Traini M, Jessup W. Lipid droplets and adipose metabolism: a novel role for FSP27/CIDEC. Curr Opin Lipidol. 2009; 20:147-149.

35. Oort PJ, Warden CH, Baumann TK, Knotts TA, Adams SH. Characterization of Tusc5, an adipocyte gene co-expressed in peripheral neurons. Mol Cell Endocrin. 2007; 276:24-35.

36. Koide H, Shibata T, Yamada N, Asaki T, Nagao T, Yoshida T, Noguchi Y, Tanaka T, Saito Y, Tatsuno I. Tumor suppressor candidate 5 (TUSC5) is expressed in brown adipocytes. Biochem Biophys Res Commun. 2007; 360:139-145.

37. Knotts TA, Lee HW, Kim JB, Oort PJ, McPherson R, Dent R, Tachibana K, Doi T, Yu S, Reddy JK. Molecular Characterization of the Tumor Suppressor Candidate 5 Gene: Regulation by PPAR and Identification of TUSC5 Coding Variants in Lean and Obese Humans. PPAR Res. 2010; 2009:13.

38. Rana A, Yen M, Sadaghiani AM, Malmersjö S, Park CY, Dolmetsch RE, Lewis RS. Alternative splicing converts STIM2 from an activator to an inhibitor of store-operated calcium channels. J Cell Biol. 2015; 209:653-670.

39. Liang L, Mujun Z, Zhenhua X, Yokoyama KK, Tsaiping L. Molecular cloning and characterization of CIDE-3, a novel member of the cell-death-inducing DNA-fragmentationfactor (DFF45)-like effector family. Biochem J. 2003; 370:195-203.

40. Inohara $\mathrm{N}$, Koseki $\mathrm{T}$, Chen $\mathrm{S}, \mathrm{Wu} \mathrm{X}$, Núñez G. CIDE, a novel family of cell death activators with homology to the $45 \mathrm{kDa}$ subunit of the DNA fragmentation factor. EMBO J. 1998; 17:2526-2533.

41. Li H, Chen A, Shu L, Yu X, Gan L, Zhou L, Yang Z. Translocation of CIDEC in hepatocytes depends on fatty acids. Genes Cells. 2014; 19:793-802. 
42. Li M, Sun X, Zhou Y, Wei X, Sun Y, Lan X, Lei C, Chen H. Nicotinamide and resveratrol regulate bovine adipogenesis through a SIRT1-dependent mechanism. J Funct Foods. 2015; 18:492-500.

43. Registre M, Goetz JG, Pierre PS, Pang H, Lagacé M, Bouvier M, Le PU, Nabi IR. The gene product of the gp78/AMFR ubiquitin E3 ligase cDNA is selectively recognized by the $3 \mathrm{~F} 3 \mathrm{~A}$ antibody within a subdomain of the endoplasmic reticulum. Biochem Biophys Res Commun. 2004; 320:1316-1322.
44. Nakamura N, Rabouille C, Watson R, Nilsson T, Hui N, Slusarewicz P, Kreis TE, Warren G. Characterization of a cis-Golgi matrix protein, GM130. J Cell Biol. 1995; 131:1715-1726.

45. Kondo S, Al-Hasani H, Hoerder-Suabedissen A, Wang WZ, Molnár Z. Secretory function in subplate neurons during cortical development. Front Neurosci. 2015; 9:100. 